\section{Systemic Sclerosis after Bone Marrow Transplantation: A Case with Evidence of Donor-related Disease Transfer}

\section{To the Editor:}

A 34-year-old man was diagnosed in January 2002 with acute B lymphoblastic leukemia. After induction chemotherapy with vincristine and daunorubicin, complete remission was achieved. In October 2011 a bone marrow relapse was detected. He was treated with clofarabine, attaining morphological remission with minimal residual disease, and in February 2012 he received a haplo-identical allogeneic bone marrow transplantation (BMT) from his mother, achieving a second complete remission. Graft versus host disease (GVHD) prophylaxis consisted of cyclophosphamide on days +3 and +4 , short-term mycophenolate mofetil, and cyclosporine as maintenance therapy. Complete donor chimerism was seen from Day +30 and has been maintained since.

Ninety days after BMT, he developed a maculopapular rash with $>50 \%$ of the skin affected, diagnosed as acute GVHD grade II. He required prolonged corticosteroid treatment until clinical resolution. In March 2013 he presented with telangiectasia on his face, forearms, and abdomen, and dry eye and dry mouth that were attributed to chronic GVHD. Only artificial tears were added to the treatment with cyclosporine. In November 2016 a digital ulcer appeared on the index finger of his left hand. He was treated with nifedipine $5 \mathrm{mg}$ twice a day and topical tacrolimus and bethametasone, without improvement.

On his first rheumatology visit in November 2017 he presented with 2 necrotic and 2 cicatricial ulcers (Figure 1), and telangiectasia on his face and neckline. Skin thickening was not observed but he complained about Raynaud phenomenon (RP) since the BMT.

He had positive antinuclear antibodies (ANA) at a titer of 1/640 with centromere pattern, anticentromere antibodies (ACA), rheumatoid factor (RF; $652 \mathrm{IU} / \mathrm{ml}$ ), and a mild consumption of C3 (86.4 mg/dl). Prior to BMT, his ANA status was negative. On the nailfold videocapillaroscopy (NVC) we observed an active scleroderma pattern, with frequent giant capillaries, microhemorrhages and moderate loss of capillaries $^{1}$ (Figure 2). He exhibited mild dysphagia, but his decision was for organ involvement not to be studied. He fulfilled the 2013 systemic sclerosis (SSc) American College of Rheumatology (ACR)/European League Against Rheumatism (EULAR) diagnostic criteria ${ }^{2}$ on the basis of digital ulcers, pitting scars, telangiectasia, abnormal nailfold capillaries, RP, and positive ACA.

He had no familial background of connective tissue diseases, but his mother had RP since her youth. On physical examination she had facial telangiectasia, sclerodactyly (modified Rodnan skin score of 4), and fingertip pitting scars, for which she had never sought treatment. She had positive ANA at titers of 1/320 with centromere pattern, ACA, RF (159 $\mathrm{IU} / \mathrm{ml}$ ), and complement consumption $(\mathrm{C} 3=74.3 \mathrm{mg} / \mathrm{dl}, \mathrm{C} 4=7.6 \mathrm{mg} / \mathrm{dl})$. On the NVC we observed a late scleroderma pattern, with severe loss of capillaries, disorganization of the normal capillary array, and ramified/bushy capillaries $^{1}$. She did not present symptoms of organ involvement. She was diagnosed with limited cutaneous SSc and fulfilled the 2013 ACR/EULAR diagnostic criteria ${ }^{2}$ on the basis of sclerodactyly, pitting scars, telangiectasia, abnormal nailfold capillaries, RP, and positive ACA.

Ethics board approval was obtained for publication and diffusion of this case report from the Ethical Committee of the Hospital General Universitario Gregorio Marañón, and both patients provided informed consent according to the Declaration of Helsinki.

This report describes a man with acute B lymphoblastic leukemia in whom SSc developed after BMT from his mother. The autoimmunity post-BMT can be due to various mechanisms ${ }^{3}$. In our case, the main difficulty is to discriminate autoimmune disease from chronic GVHD. For many autoimmune disorders, adoptive transfer of diseases from the donor to the recipient after BMT has been documented ${ }^{3,4}$. It is known that the hematopoietic and immune system of recipients is eventually replaced by donor-derived cells after allogeneic BMT. Because of that, hematopoietic stem cell donation is generally not recommended in individuals with autoimmune systemic disorders ${ }^{5}$. In this case, we find evidence that suggest a donor-related disease transfer: the absence of previous manifestations and previously negative autoantibodies, the typical NVC images, the presence of digital ulcerations and RP that is exceptional in $\mathrm{GVHD}^{6}$, the presence of ACA (which has never been described among the autoantibodies found in chronic GVHD) ${ }^{7,8}$, and most importantly, the donor being diagnosed with SSc. Although all these data suggest a donor origin, we cannot completely exclude the possibility of chronic GVHD. This hypothesis is supported by the patient's acute GVHD, which may also lead to nailfold capillary abnormalities ${ }^{9}$; in addition, we cannot demonstrate visceral involvement typical of SSc. This could be a case of adoptive transfer of SSc from the donor to the recipient after BMT.

ALICIA SILVA RIVEIRO (D) , MD; JUAN CARLOS NIETO GONZÁLEZ, MD, PhD; JULIA MARTÍNEZ BARRIO, MD, PhD, Department of Rheumatology, Hospital General Universitario Gregorio Marañón, Madrid, Spain. Address correspondence to Dr. A. Silva Riveiro, Department of Rheumatology, Hospital General Universitario Gregorio Marañón, Calle del Doctor Esquerdo 46, 28009, Madrid, Spain.

E-mail: alicia.silvariveiro@gmail.com

\section{REFERENCES}

1. Cutolo M, Sulli A, Pizzorni C, Accardo S. Nailfold videocapillaroscopy assessment of microvascular damage in systemic sclerosis. J Rheumatol 2000;27:155-60.

2. van den Hoogen F, Khanna D, Fransen J, Johnson SR, Baron M, Tyndall A, et al. 2013 classification criteria for systemic sclerosis: an American College of Rheumatology/European League against Rheumatism collaborative initiative. Arthritis Rheum 2013;65:2737-47.

3. Sherer Y, Shoenfeld Y. Autoimmune diseases and autoimmunity post-bone marrow transplantation. Bone Marrow Transplant 1998;22:873-81.

4. Snowden JA, Brooks PM, Biggs JC. Haemopoietic stem cell transplantation for autoimmune diseases. Br J Haematol 1997; 99:9-22.

5. Hough R, Snowden J, Wulffraat N. Haemopoietic stem cell transplantation in autoimmune diseases: a European perspective. $\mathrm{Br}$ J Haematol 2005;128:432-59.

6. Ferreli C, Gasparini G, Parodi A, Cozzani E, Rongioletti F, Atzori L. Cutaneous manifestations of scleroderma and scleroderma-like disorders: a comprehensive review. Clin Rev Allergy Immunol 2017;53:306-36.

7. Rouquette-Gally A, Boyeldieu D, Prost A, Gluckman E. Autoimmunity after allogeneic bone marrow transplantation. A study of 53 long-term-surviving patients. Transplantation 1988;46:238-40.

8. Bell S, Faust H, Mittermüller J, Kolb H, Meurer M. Specificity of antinuclear antibodies in scleroderma-like chronic graft-versus-host disease: clinical correlation and histocompatibility locus antigen association. Br J Dermatol 1996;134:848-54.

9. Akay B, Sanli H, Topcuoglu P, Arat M, Akyol A. Nailfold capillary abnormalities are prevalent in sclerodermoid graft-versus-host disease and readily detected with dermatoscopy. Br J Dermatol 2010;162:1076-82.

First Release May 15 2019; J Rheumatol 2019;46:866-6; doi:10.3899/jrheum.180853 


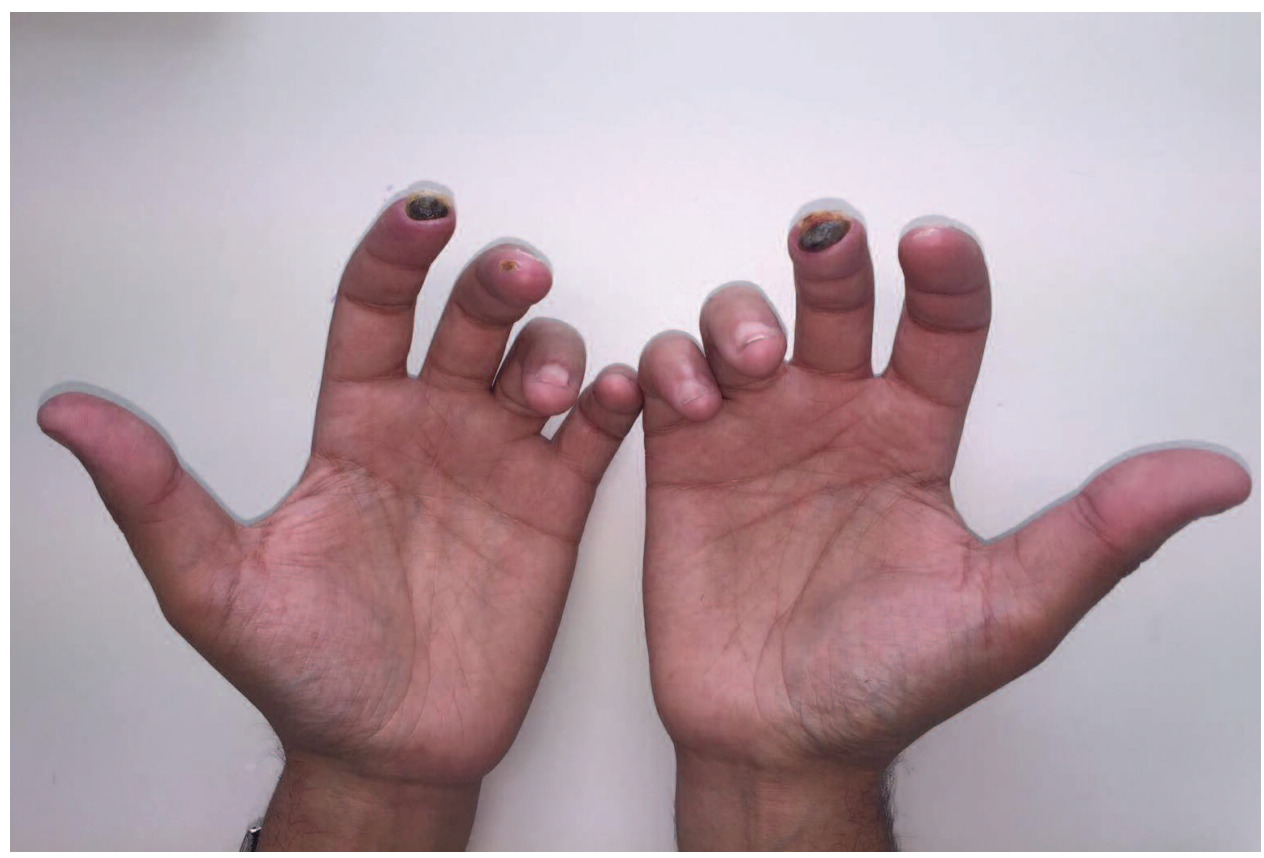

Figure 1. Necrotic ulcers on the index finger of the left hand and on the middle finger of the right hand. Cicatricial ulcers on the middle finger of the left hand and on the index finger of the right hand.

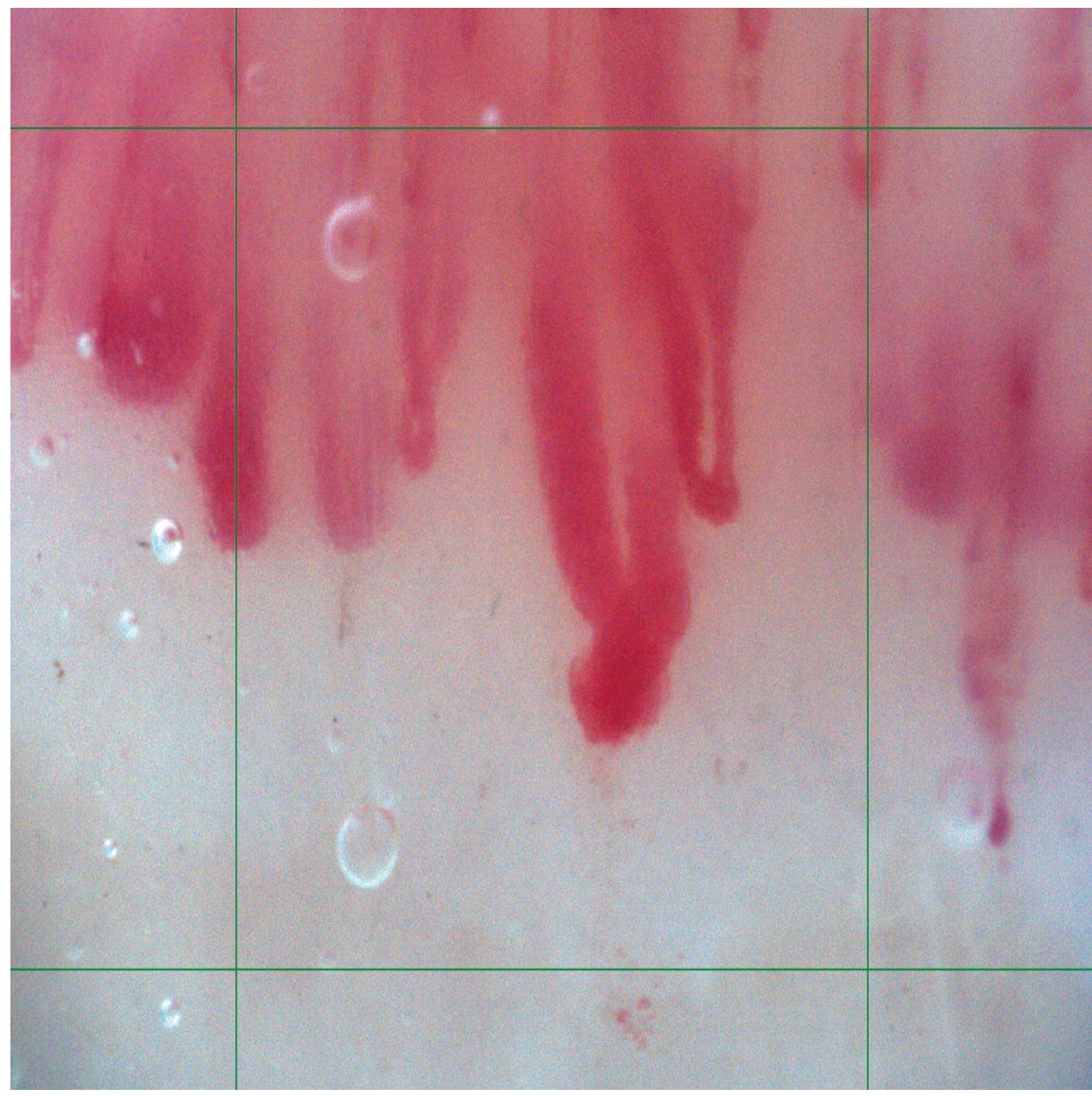

Figure 2. Image of nailfold videocapillaroscopy. Moderate loss of capillaries and a giant capillary with remains of a microhemorrhage. 